\title{
What if Burnout was a Symptom of Our 21st Century Society?
}

\author{
Andrée Piecq \\ Master in Psychology, General Secretary UES-EUS, Honorary President S \& O, Scientific Director G.I.R.O.S (Belgium), \\ Rue Beauregard 98, Carnières, B-7141, Belgium
}

Copyright $(\mathrm{O} 2018$ by authors, all rights reserved. Authors agree that this article remains permanently open access under the terms of the Creative Commons Attribution License 4.0 International License

\begin{abstract}
To verify the hypothesis that burnout is a symptom of the 21 st century, this article analyzes burnout in organizations. Burnout in organizations is studied by: The evaluation of the organization's functioning; the diagnosis of the organization as "operative" or as "inoperative" in order to choose steering strategies. Our society and the organizations are considered as isomorphic structures. The methodology used here is the "Giroscope"[1] and the "12 guiding principles [1]" which are two patterns used to analyze human organizations. General explanation about the "Giroscope" analysis: When the target is to make an evaluation of the functioning of an organization: The contexts are considered and the " 12 guiding principles" are used; The organization's diagnosis gives the opportunity to characterize it as "operative" or "inoperative"; This diagnosis is made to allow the development of steering strategies in order to enable the modification of the organization; To introduce modifications in "operative" organizations, the structure must not be changed. However, in an "inoperative" organization, it is necessary to change the structure. The hypothesis of this article is that organizations, in which burnout emerges, have their structure diagnosed as "inoperative". The necessary strategy to change this structure into an "operative" one is "the mutation".
\end{abstract}

Keywords Burnout, Guiding Principles, Complexity, Context, Border, Finality, Feedback, Isomorph

\section{Introduction}

"Burnout" has always existed and can, in some cases, lead human beings either to "die" when it takes the upper hand or to surpass it thanks to resilient behaviors [2].

Generally, burnout is considered as an employee's physical and mental disease. The diagnostic and the treatment of burnout are the taken in charge by doctors, psychotherapists or coaches.
For "the Independent Institute of the Systemic of Organizations (G.I.R.O.S)", burnout is a symptom of the dysfunction of organizations. It first alerts the managers and then pushes them to analyze the functioning of their organization.

The "Giroscope" model falls within the framework of systemic epistemology. Its particularity consists in putting systemic concepts together in order to evaluate the functioning of systems' structures and more specifically the functioning of human systems' structures (market institutions or non-profit institutions). These systemic elements are concepts and they are called the "12 guiding Principles ". They interact with one another that is to say they are in relations. These relations are correlated to form a coherent and complex unit: a system. The observation of the guiding principles provides access to the system's structure which enables the diagnosis of the system's functioning.

The evaluation of the systems has two different objectives:

- The understanding of a systems' functioning ;

- The establishment of the necessary diagnostic to pilot a human organization.

\section{Organizations in Which Burnout Appears}

\subsection{Their Context}

Since the last century, one of the contexts inherent to organizations is linked to our societal context where productivity and profit must be at maximum. Members are asked to evolve and to change constantly. Time runs away too quickly, there is no time to metabolize change, phases of homeostasis miss, everything must be done urgently and the qualifications and performances of members are constantly optimized.

Another important element is the emergence of more and more advanced technologies. 
All these contexts added to the context of professional qualification weigh on organizations. Moreover, escaping from these organizations in order to survive is almost impossible. It is as if this paradoxical situation and the frantic pace of change could only lead to the burnout of employees and finally lead to the collapsing of the organization.

\subsection{The "12 Guiding Principles"}

The methodology based on the "guiding principles" is an emergence of the concepts studied by Gregory Bateson [3], Ludwig von Bertalanffy [4], Heinz von Forester [5], Francisco Varela [6], Humberto Maturala [6] Paul Watzlawick [3], , Joël de Rosnay,[7] Ilya Prigogine [8], Mony Elkaïm [9], (this list is not exhaustive). They are: system, totality, and emission of information, reception of information, feedback, finality, "equifinality", borders, members, rules, circularity, and homeostasis.

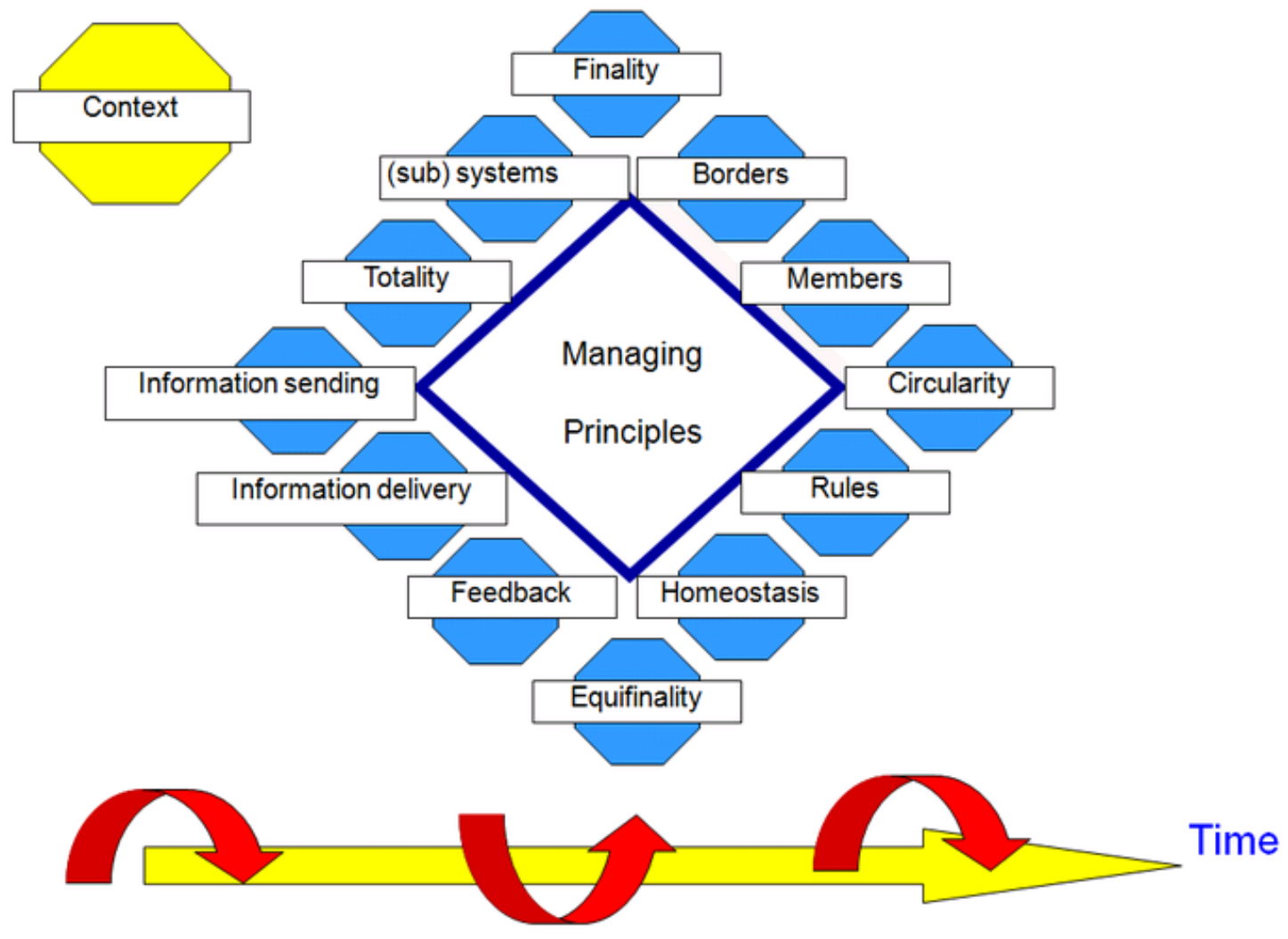

Figure 1. The 12 Guiding principles

The "Giroscope" model is not only theoretical, it is also operative. It has been used for more than twenty years, to help different types of organizations.

The 12 "guiding principles" are concepts and are thus abstract. In order to act it is necessary to have concrete material. This is only possible by observing repetition of member's behaviors.

Observing the behaviors between members, informs not only about their direct interactions but also about the interactions existing between subsystems and between the organization and its context.

A single study of each individual "guiding principle" gives a lot of information but it is not sufficient. The observation of interactions is essential to diagnose the functioning of the company (the organization). In this way, we can observe that guiding principles interact with one another.

In this paper, only four principles are chosen and are presented as a sample. These four principles are the borders [1], the feedback [1] the finality [1] and the "equifinality" [1].

\subsubsection{The Borders}

Borders are the junction between the context and the organization, between the organization and its subsystems, between members and subsystems, and between members together. Borders are in close interaction with

- the finality principle (the purpose, the objectives and the actions of the organization, departments and members)

- the information's reception and emission principle, (how the information is transmitted and received): 
- If the information reach their target [1]: the borders are flexible;

- If no information are transmitted [1]:the borders are rigid;

- If the information overwhelms the organization [1]: the borders are diluted.

In the context described above, the exchange of information with the outside is permanent and labile. It is as if the information had to be treated immediately, and should quickly lead to the implementation of novelty (changes of all kinds). Sub-systems are undifferentiated; hierarchies are no longer respected [10].

The borders are too porous and diluted.

As a consequence, the elements of information will drown the organization. Borders will not be there as a filter. The main risk is that the organization will lose its identity and its finality. Assimilating and managing all the elements of information becomes a desperate attempt. Subsystems (departments) and members of the organization are overloaded and in burnout.

\subsubsection{The Finality}

Finality is divided into 3 levels: the main goal, the objectives and the actions [1]. These three levels:

- Describe what the organization, its sub-systems and its members, want to do, how and when;

- Highlight how the members of the organization must interact;

- Happen at different times: long, medium and short-term;

- Are interdependent and are the three necessary steps which lead to the organization's evolution;

- $\quad$ Are in interaction with all the guiding principles [1]

If these three levels do not exist, or if they are not well defined, the finality of the organization is undefinable. The main goal, the objectives and the actions are elusive, mixed, unclear and imprecise.

Observations of the functioning of the company show that time goes by too quickly, it is uncontrolled, everything goes too rapidly which leads to the implementation of more and more changes. These changes may endanger the finality of the organization. Indeed the three levels are no longer differentiated in time. In this context, how could it be possible to assess the impact of past actions and to adapt future actions when past, present, and future happen at the same moment? The short, medium, and long-term are gathered in one single time: the immediate time.

The immediate time cancels the finality sequences. The sequences are no longer logical: the main goal becomes actions, actions become objectives and objectives become the main goal. As a result, the organization becomes "inoperative".

Actions are taken before knowing the how and why of things.

Individual finalities (from members or subsystems) are in conflict or are at variance with those of the organization; they are neither clear nor marked: the levels and the hierarchies are mixed.

The gap between the official finality and reality generates nonsense, causing the exhaustion of human resources.

When burnout emerges, the observation of member's behavior shows that individualization and empowerment are impossible. Members no longer know, or no longer understand, when they have to act. The result is the impossibility for them to find their place in regard to the organizational finality.

Doing better, more and faster, forgetting the three finality levels (main goal, objectives, actions), opening the borders, letting all information enter, it is as if the chaos settles.

\subsubsection{The Feedback [11]}

In organizations, to verify that an action has reached its goal, it is necessary to collect pieces of information. Such elements form "a closed loop" which gives the opportunity to evaluate if the performances of past actions [12] are appropriate for future actions. This loop is the "feedback" [1]. The feedback mechanisms interact with all the guiding principles and take an important place in the functioning of the organization:

- Without them, no checking is possible;

- They bring back the organization to a stability, to a direction;

- They guarantee self-preservation;

- They generate adaptive behaviors because they bring the system back to a point of equilibrium;

- They allow a break in the race to change;

- They impulse change

Two sorts of feedbacks are considered:

- The negative feedback loop corrects the disturbances of the organization and restores the original equilibrium.

- The positive feedback impulses changes

The observation of organizations in which burnout appears shows that the orientation -the goal- of the action is lost, forgotten or even ignored. There are no more feedback loops (nor positive, nor negative). This absence of correction (feedback) leads to members' insecurity, because it is impossible for them to know if they have reached the desired result.

It shows that the absence of "feedback", positive as well as negative, leads to imbalance.

Moreover, reaching equilibrium is impossible because everything goes too fast. Too few negative feedbacks appear. To change is the major rule.

This is the realm of positive feedback. It is as if the time gets shorter and as if there was only one temporal sequence: the immediate.

\subsubsection{The "Equifinality"}

"Equifinality" completes the notion of finality. Ludwig 
von Bertalanffy [12] has highlighted it. "Equifinality" is one of the characteristics of interacting systems (systems made up of living elements).

Indeed, it is the structure of open systems that determines the consequences (the results) of the processes involved and not the initial conditions they were in.

That means that if two systems have the same structure (the same types of interactions between PDIs), they can produce, or lead to the same result. They are thus called isomorphic.

Although the concrete form of the "guiding principles" governing our Societies is at a higher level of complexity than in an ordinary organization, the interactions between their guiding principles are of the same type.

A valid hypothesis is that these two systems are isomorphic and so they have the same functioning. Moreover, it is possible to consider organizations as subsystems of the "Society" system: they have recurrent features and the same structure. By analogy, they are called fractal (discovered by Benoit Mandelbrot (1975)

\section{Conclusions}

In this article, burnout is considered as a symptom of the 21st century: the system (society) and the subsystems (organizations) are isomorphic; they have the same structure and are malfunctioning.

The societal cost of the burnout is enormous [13]; following this theoretical demonstration concerning its emergence, a practical conclusion is necessary. This conclusion is a brief description of how the systemic model "The Giroscope" [1] is used by the G.I.R.O.S' advisers and by some managers.

Difficulties, inconsistencies within "guiding principles" and within interactions lead to burnout. Two strategic choices exist to avoid it:

- The "Cosmetic" modifications [1] which implementation modifies the contents of the "guiding principles". Cosmetic modifications are implemented in the medium and short-term. They are based on internal resources in organization and are purely corrective actions (the organization remains the same; its structure is not changed). Organizations face logically surmountable situations by resorting to common sense.

When introducing change, it is an error to assume the fact that as the previous actions were not suitable; the opposite actions are the right one to choose.

- The "Structural" changes [1], the "mutation" which implementation is in the long-term (by working on the interactions between "guiding principles"). It requires a transformation of the structure of the organization. (Common sense only is not enough to solve dead ends or inextricable situations.)

The implementation of the two strategies is totally different, even if the gates to install the "cosmetic" change and the "structural" change are the same:

1. Observing members' behaviors and considering these behaviors as a symptom of the malfunctioning of the organization, as a warning signal of the difficulties of the organizations

2. Elaborating hypothesis about "guiding principles" that have been impacted by the observed behaviors;

3. Observing the interactions between the elements of the system with one another and between the elements and the contexts.

4. Making the diagnostic with the 12 "guiding principles" (finality, borders, rules, feedback, communications...).

5. Developing strategies for change: a "cosmetic" approach or a structural strategy.

A structural strategy modifies the structure of organizations by changing the interactions between the "guiding principles" (the order, the ratios they have...).

When burnout is installed, the only right strategy is to change the structure of the organization by modifying the interactions between the "guiding principles".

Introducing this total change in the structure in the short or medium term is almost impossible for companies. It could totally destroy them because of the high costs it implies in terms of time, money, management...

For the Society, this kind of change would cost a lot of troubles and crisis.

The way to introduce this change is to go one step at a time. In the case of burnout, the diagnostic highlighted problems within "guiding principles" (see upper paragraphs)

One example of strategy consists in pushing managers of the organization to introduce modifications in:

The finality: specify and clarify the objectives, the goals, the actions, the missions, the roles, the complementarities and the hierarchical links.

The feedbacks: verify that explanations are given when new processes are set up to motivate members and to give them pieces of information about what they are doing, in order to accept the change.

The borders: clarify the limits not to transgress between the different missions, actions and complementarities and also between the members and the hierarchies.

The rules: explain them and in some cases modify them; removing the implicit rules and making them as explicit as possible.

As a conclusion, the best way to avoid burnout is to establish a prevention system by implementing "circular evaluation" [1] in order to make a constant evaluation of the functioning of the organizations with the "guiding principles".

The "circular evaluation" is a circular process [14]. It relies on the "Giroscope" model to decode the functioning of the organization and to escape the risk that the organization becomes, at one time, dysfunctional and generates burnout. 
The circular evaluation can be used to stop the negative spiral.

The same process could also be implemented within our society, but is it realistic in the current world's political context?

\section{REFERENCES}

[1] A.Piecq. De la Pensée Systémique à la Pratique de l'Organisation : Le Giroscope, L'Harmattan, Paris 2011,pgs $113,143,144,131,137,125,141,138,139,177$ to 190,216 to 219

[2] G..Koninckx , G.,Teneau., « Résilience organisationnelle Rebondir face aux turbulences », de boeck, 2010.

[3] P.Watzlawick, Beavin J-H., Jackson D.D.,’Une logique de la communication”, Le Seuil ,1972

[4] L.Von Bertalanffy, "La Théorie Générale de systèmes », Dunod, Paris 1973

[5] H.Von Foerster, Observing Systems, Intersystems Publications, Seaside, CA, 1981
[6] F. Maturana, F.Varela. Autopiesis and Cognition: the Realization of the Living. Boston Studies in the Philosophy of Sciences, vol. 42, Reidel 1980.

[7] J De Rosnay. L'homme symbiotique, Le Seuil 1995.

[8] I. Prigogine. L'homme devant l'incertain. Odile Jacob, 2001.

[9] M.Elkaïm. Si tu m'aimes, ne m'aimes pas, Le Seuil, 1989.

[10] P. Watzlawick, J. H Weakland. Sur l'interaction, Le Seuil 1981.

[11] N.Wiener, Cibernétique et Société, Union Générale d'Edition. Christian Bourgeois-Dominique De Roux. Coll. 10/18 1971 .

[12] L. Bertalanffy , "La Théorie Générale de systèmes », Dunod, Paris 1973

[13] Information letter from Social secretariat Securex., Cost for INAMI (National Institute for Sickness and Invalidity Insurance): 600 million euros per year - the number of employees at risk of burnout has increased from $10 \%$ in 2014 to $17 \%$ in 2017. 9 September 2016

[14] W.R. Ashby, « An Introduction to Cybenetics », Chapman \& Hall, London, 1956 